\title{
Hyperhemolysis Syndrome Following Red Cell Exchange in a Newly Diagnosed Sickle Cell Disease Patient With Spinal Cord Infarction
}

\author{
David Starr ${ }^{\mathrm{a}}$, Daniela Hermelin ${ }^{\mathrm{a}}$, Douglas Blackall ${ }^{\mathrm{a}, \mathrm{b}}$
}

\begin{abstract}
Hyperhemolysis syndrome (HS) is a rare red blood cell (RBC) transfusion reaction that shares similarities with other hemolytic transfusion reactions. Because of this, it is important to recognize key presenting clinical and laboratory features in order to guide therapy. In this case report, a patient with a sickling hemoglobinopathy who developed HS is presented. The atypical nature of this case resides in the clinical presentation of paraplegia secondary to spinal cord infarction, increasingly complex blood group serological findings, and multiple RBC exchanges prior to the HS reaction. Once the patient was diagnosed with probable HS, approximately 4 weeks into her clinical course, RBC transfusion (including exchange transfusion) was withheld. Instead, corticosteroids and erythropoietin were initiated without complication. The patient remained stable with this treatment modality until her care was transferred to a hospital with a comprehensive sickle cell center. This case highlights the need to withhold transfusion in HS patients, barring exceptional circumstances, and the efficacy of initiating immunomodulatory and erythropoiesis stimulating therapies.
\end{abstract}

Keywords: Blood transfusion; Exchange transfusion; Hemolysis; Sickle cell disease; Sickling hemoglobinopathy; Transfusion reaction

\section{Introduction}

Sickle cell disease (SCD) is an autosomal recessive red blood cell $(\mathrm{RBC})$ disorder in which the beta-globin subunit of hemoglobin $(\mathrm{Hb}) \mathrm{A}$ is replaced with $\mathrm{HbS}$ [1]. SCD affects approximately 4.4 million people worldwide, with more than 300 million people having sickle trait $[1,2]$. The critical patho-

Manuscript submitted July 3, 2020, accepted July 10, 2020

Published online August 6, 2020

aDepartment of Pathology, Saint Louis University School of Medicine, Saint Louis, MO 63104, USA

${ }^{b}$ Corresponding Author: Douglas Blackall, Department of Pathology, Saint Louis University School of Medicine, 1402 South Grand Blvd., Saint Louis, MO 63104, USA. Email: Douglas.Blackall@health.slu.edu

doi: https://doi.org/10.14740/jmc3541 physiologic disruption underlying SCD occurs in low oxygen tension environments in which the normal biconcave shape of the RBC is transformed into a sickle shape [1]. This shape change prevents appropriate oxygen delivery and can lead to a sequalae of problems related to poor oxygenation of tissue and oxidative stress. This promotes downstream effects such as vaso-occlusive crises, $\mathrm{RBC}$ destruction leading to anemia, and immune dysfunction increasing the risk for infections [1]. First-line therapy for SCD is directed to preventing vaso-occlusive episodes [1]. There are a variety of treatment options that are pursued in the clinical setting, the most common of which are blood transfusion, hydroxyurea administration, and blood marrow transplantation.

Despite recent advances in SCD treatment (e.g., disease modifying therapeutics and gene therapy approaches), there continues to be many challenges for the patient and the medical system in terms of quality of life and prognosis. A significant hurdle in treating SCD patients relates to blood group antibody formation due to recurring blood transfusions [3, 4]. Given that these patients are likely to require multiple blood transfusions in their lifetime and that they are at increased risk for developing complications related to blood transfusion, it is important to understand the clinical presentation of these complications and their treatment. Hyperhemolysis syndrome (HS) is the focus of this paper since it is atypical in its presentation and requires a unique therapeutic approach unlike those provided in the context of other transfusion-related complications.

\section{Case Report}

A 36-year-old African American woman presented to the emergency department for lower extremity weakness, urinary and stool retention, and a burning sensation on her abdominal skin. The patient had type II diabetes mellitus but was otherwise well. She had a history of RBC transfusion in association with the treatment of HELLP syndrome several years before presentation. Her physical examination revealed decreased lower extremity strength, a decreased sensation to temperature and pin prick below dermatome T5, proprioception preservation, and no involvement of the cranial nerves. In addition, there were no abnormalities of her vital signs. Laboratory testing revealed an $\mathrm{Hb}$ of $9.5 \mathrm{~g} / \mathrm{dL}$ (decreased from a baseline of $13.1 \mathrm{~g} /$ $\mathrm{dL}$ ), a hematocrit (Hct) of $27.9 \%$ (baseline of $38.3 \%$ ), a white 
blood cell count of $3.8 \times 10^{9} / \mathrm{L}$, and a platelet count of 131,000 $\times 10^{9} / \mathrm{L}$. Her serum glucose was $168 \mathrm{mg} / \mathrm{dL}$, and her HbA1c was $5.7 \%$. Magnetic resonance imaging (MRI) of the thoracic and lumbar spine demonstrated a bilateral, symmetric, nonenhancing central spinal cord signal abnormality, from T6 - 7 through T9 - 10, suspicious for spinal cord infarction. Because of this unusual finding, a sickling hemoglobinopathy was considered in the differential diagnosis. To that end, $\mathrm{Hb}$ electrophoresis testing was performed and demonstrated $71.4 \%$ $\mathrm{HbS}, 19.0 \% \mathrm{HbA}, 6.3 \% \mathrm{HbA}_{2}$, and $3.7 \% \mathrm{HbF}$ consistent with a diagnosis of $\mathrm{HbS} / \mathrm{B}^{+}$thalassemia. Hematology-Oncology was consulted and recommended emergency RBC exchange. Initial blood bank serological testing revealed that the patient was A-positive and had an anti-E blood group antibody. Nine units of group- and crossmatch-compatible $\mathrm{RBC}$ units were provided that were $\mathrm{Hb}$ S-negative and lacked the $\mathrm{C}$, E, and $\mathrm{K}$ blood group antigens. The patient tolerated the procedure well and had no complications. The following day, her $\mathrm{Hb}$ was $10.3 \mathrm{~g} / \mathrm{dL}$, and her $\mathrm{HbS}$ had decreased from $71.4 \%$ to $18 \%$. The patient's clinical course was stable. She continued as an inpatient and engaged in rehabilitation activities. The immediate medical plan was to repeat RBC exchanges, as necessary, to maintain her $\mathrm{HbS}$ below $30 \%$.

Approximately 2 weeks after admission, the patient underwent a second $\mathrm{RBC}$ exchange as her $\mathrm{HbS}$ had increased to $41.5 \%$. Her $\mathrm{Hb}$ had also fallen to $8.0 \mathrm{~g} / \mathrm{dL}$. Blood bank testing demonstrated a strongly reactive antibody screen with anti-E, anti-Fy ${ }^{\mathrm{b}}$, anti-Jk ${ }^{\mathrm{b}}$, anti-C, and anti-K antibodies detected in her plasma. However, her direct antiglobulin test (DAT) was negative. The exchange transfusion was undertaken with six crossmatch-compatible units of blood lacking the $\mathrm{E}, \mathrm{Fy}^{\mathrm{b}}, \mathrm{Jk}^{\mathrm{b}}$, $\mathrm{C}$, and $\mathrm{K}$ antigens. The patient tolerated the procedure well and had no immediate complications. Her post-exchange labs revealed a $\mathrm{Hb}$ of $9.6 \mathrm{~g} / \mathrm{dL}$ and a $\mathrm{HbS}$ of $16 \%$. The patient improved clinically and was transferred to a rehabilitation facility to continue therapy for lower extremity weakness. Follow-up was arranged at a comprehensive sickle cell center for outpatient care and consideration for further RBC exchanges.

Twelve days after discharge, the patient presented to an emergency department for the evaluation of right-sided chest pain and worsening lower extremity weakness. Computed tomography (CT) of the chest demonstrated bilateral pulmonary embolisms. Her laboratory values were significant for an $\mathrm{Hb}$ of $5.7 \mathrm{~g} / \mathrm{dL}$, an Hct of $18.2 \%$, an absolute reticulocyte count of $0.14 \times 10^{9} / \mathrm{L}$ (reference interval: $0.02-0.13$ ), and an $\mathrm{HbS}$ of $64.8 \%$. Hematology-Oncology was consulted and recommended anticoagulation with heparin and RBC transfusion, with the possibility of RBC exchange. Blood bank serological testing revealed anti-E, anti-Jk ${ }^{\mathrm{b}}$, anti-C, and anti- $\mathrm{C}^{\mathrm{w}}$ alloantibodies. In addition, the patient had a newly reactive DAT with positive immunoglobin $\mathrm{G}$ (IgG) testing and negative $\mathrm{C} 3$ testing. Further testing identified a warm-reactive autoantibody with specificity for the D blood group antigen. The patient's presentation was felt to be most consistent with HS in association with a possible delayed hemolytic transfusion reaction (DHTR). Based on these findings, the patient's treatment plan transitioned to only transfuse blood products for life-threatening anemia in order to avoid additional immune stimulation. In addition, an erythropoietin-stimulating agent $(10,000 \mathrm{U} / \mathrm{mL}$ injection of epoetin alfa-epbx) was initiated along with a steroid (125 mg intravenous (IV) daily of methylprednisolone). The patient remained stable on this treatment plan and required no further transfusion prior to transfer to a hospital with a comprehensive sickle cell center.

\section{Discussion}

Patients with SCD are more likely to receive blood transfusions than the overall population. As such, they are at increased risk for transfusion reactions. In particular, patients with SCD are more prone to forming alloantibodies [3, 5-7]. There are multiple reasons for this: 1) the frequency of blood transfusions; 2) the frequency of SCD in minority patients requiring blood products from a predominantly Caucasian donor population; 3) other genetic factors [5, 6]. In addition, decreased heme oxygenase levels in SCD patients can lead to an incomplete clearance of $\mathrm{Hb}[5,6]$. Because of inefficient clearance, patients with SCD are more likely to be in a pro-inflammatory state; thus, increasing the risk of alloimmunization $[5,6]$. Specifically, it is estimated that $5-36 \%$ of SCD patients will develop antibodies to RBC antigens [8]. This is important because alloimmunization puts SCD patients at risk for complications such as DHTRs, hemolytic disease of the fetus/newborn, and HS. DHTRs and HS are estimated to occur in $1-19 \%$ of SCD patients over a lifetime [8].

HS is a rare complication of RBC transfusion that is characterized by a life-threatening post-transfusion anemia with a marked decrease in $\mathrm{Hb}$ and Hct compared to pre-transfusion levels [3, 5-7]. There is also an associated decrease in haptoglobin levels and an increase in lactate dehydrogenase (LDH) and bilirubin [5, 6]. HS can be distinguished from a DHTR as there is destruction of both transfused and autologous RBCs in HS, while DHTRs are only associated with the destruction of transfused cells [3, 5-7].

The clinical presentation of HS is varied but can mimic a variety of SCD complications including infections and vaso-occlusive crises [3, 5-7]. It is also important to note that while HS is most commonly seen in SCD patients, patients with thalassemia, myelofibrosis, anemia of chronic disease, and lymphoma are also at risk [9]. Patients with Kidd (Jk) antibodies also seem to be more susceptible to developing HS [10]. Presenting symptoms of HS include, but are not limited to, fever, fatigue, dyspnea, jaundice, hemoglobinuria, reticulocytopenia, and pain [3, 5-7]. Laboratory testing that may assist in the diagnosis of HS includes a reticulocyte count, serum bilirubin level, LDH, DAT, and Hb electrophoresis [3, $5,6,9]$. HS can be either acute or delayed. In acute HS, patients have symptoms within 7 days of transfusion. There is no associated alloantibody formation and the DAT is negative $[3,5,6,9]$. In contrast, delayed HS, as seen in this case report, is associated with alloantibody formation and a positive DAT and most commonly occurs 7 days or more after transfusion $[3,5,6]$.

There are several proposed mechanisms for the pathophysiology of HS, some of which have been thought to work in tandem. One of the most widely cited hypotheses is "by- 
stander hemolysis" $[3,5,6,10]$. This mechanism suggests that both native and donor RBCs are lysed by complement factors. Activated macrophages may also play a role in the associated hemolysis and reticulocytopenia [4, 5, 6, 10]. Sickled RBCs have more surface IgG and phosphatidylserine, which makes them susceptible to the effects of complement and macrophage activation $[6,10]$. Increased surface phosphatidylserine has also been shown to promote RBC clearance from the circulation [6]. With over-activation of macrophages, reticulocytes are susceptible to destruction as they have a surface protein, vascular cell adhesion molecule 1 (VCAM-1), which promotes their adherence to macrophages. This may contribute to reticulocytopenia [5].

Proposed treatment modalities for HS are focused on disrupting the pathophysiologic mechanisms mentioned above. The primary recommendation is that all blood transfusions should be stopped and that future transfusions should be considered carefully [4]. This is meant to reduce exacerbations and the prolongation of HS episodes. In the event of an emergency need to transfuse, there can still be complications even when ABO-matched, Rh-matched, and K-matched units of blood are transfused that are crossmatch-compatible [4-6, 9-13]. Aside from stopping transfusion, intravenous immunoglobin (IVIG) and corticosteroids, administered together or individually, are associated with the most consistent success $[3,4,9]$. The proposed mechanism for IVIG efficacy is a blocking effect that prevents the adhesion of sickled RBCs and reticulocytes to macrophages [4]. A secondary effect of IVIG is the suppression of macrophage activation through immunomodulation. Risks associated with IVIG include renal toxicity and an increased incidence of thromboembolic events [4, 6]. Corticosteroids can also suppress the activation of macrophages [4]. A current therapeutic recommendation is to provide low-dose IVIG $(0.4 \mathrm{~g} / \mathrm{kg} /$ day $)$ for 5 days and intravenous methylprednisolone $(0.5 \mathrm{~g} /$ day for adults and $4.0 \mathrm{mg} / \mathrm{kg} /$ day for children $)$ for 2 days [4].

Other treatments for HS that are in the early prospective stages of evaluation have shown promise. Eculizumab, a C5 convertase inhibitor, has demonstrated some success in preventing complement cascade activation [6, 13]. Erythropoietin promotes the stimulation of erythroid precursors and the prevention of neocytolysis [13]. Rituximab has been used successfully in severe cases of HS to promote the recovery of RBCs [3, 4, 6, 9]. Therapeutic plasma exchange has been shown to be successful in case studies of severe HS; however, the consensus is that additional study is warranted [13]. As further analysis is performed and there is improved understanding of HS, there is an expectation that early intervention and treatment will become standardized and optimized.

The clinical course of our patient is representative of the many unique challenges that can arise when taking care of SCD patients. The initial presentation of thromboembolic infarction affecting the central spinal cord combined with significant anemia suggested the possibility of an underlying sickling hemoglobinopathy $[14,15]$. When this was confirmed, an $\mathrm{RBC}$ exchange commenced. Initially, an E antibody alone was detected on antibody screening, which likely resulted from transfusion during a previous hospital admission. In the second $\mathrm{RBC}$ exchange, additional antibodies were detected, including
anti-Jk ${ }^{b}$. When the patient was discharged from her first hospital admission, there was no evidence of transfusion-related complications. The patient appeared to be responding favorably with respect to her post-exchange $\mathrm{Hb}$ and $\mathrm{HbS}$ levels. It was not until 2 weeks after discharge, when the patient was undergoing rehabilitation, that complications from transfusion became evident.

When the patient presented to the hospital a second time, there was significant anemia. Four blood group antibodies were detected (anti-E, anti-C, anti-Jk ${ }^{b}$, and anti- $\mathrm{C}^{\mathrm{w}}$ ). The patient's post-transfusion $\mathrm{Hb}$ was significantly lower than her pre-transfusion level and her DAT was positive, which suggested the possibility of an immune-mediated hemolytic process. The detection of a new warm-reactive autoantibody provides further support for this hypothesis. Finally, the significant rise of the patient's native HbS level suggests a DHTR due to the preferential destruction of transfused donor red cells, but this does not preclude that autologous cells were also destroyed (i.e., HS).

With the overlapping mechanisms of HS and DHTR in play, RBC exchange was withheld, immunomodulatory therapy was started, and erythropoietin was initiated. With this therapeutic approach, the patient was clinically stable during the remainder of her hospital course. Her $\mathrm{Hb}$ and Hct were beginning to trend upwards, and she continued to do well in an outside hospital associated with a comprehensive sickle cell center.

\section{Acknowledgments}

None to declare.

\section{Financial Disclosure}

None to declare.

\section{Conflict of Interest}

The authors have no relevant financial interests or other conflict of interest to disclose.

\section{Informed Consent}

Not applicable.

\section{Author Contributions}

David Starr participated in manuscript planning, wrote the original draft of the manuscript, revised and edited the manuscript prior to submission. Daniela Hermelin participated in manuscript planning, revised the original manuscript, and edited subsequent versions of the manuscript prior to submission. Douglas Blackall conceived of the manuscript, revised the 
original manuscript, revised and edited the manuscript prior to submission.

\section{Data Availability}

The authors declare that data supporting the findings of this study are available within the article.

\section{References}

1. Piel FB, Steinberg MH, Rees DC. Sickle cell disease. N Engl J Med. 2017;376(16):1561-1573.

2. Tsaras G, Owusu-Ansah A, Boateng FO, Amoateng-Adjepong Y. Complications associated with sickle cell trait: a brief narrative review. Am J Med. 2009;122(6):507512.

3. Santos B, Portugal R, Nogueira C, Loureiro M. Hyperhemolysis syndrome in patients with sickle cell anemia: report of three cases. Transfusion. 2015;55(6 Pt 2):13941398.

4. Win N. Hyperhemolysis syndrome in sickle cell disease. Expert Rev Hematol. 2009;2(2):111-115.

5. Aragona E, Kelly MJ. Hyperhemolysis in sickle cell disease. J Pediatr Hematol Oncol. 2014;36(1):e54-56.

6. Banks M, Shikle J. Hyperhemolysis syndrome in patients with sickle cell disease. Arch Pathol Lab Med. 2018;142(11):1425-1427.

7. Eberly LA, Osman D, Collins NP. Hyperhemolysis syndrome without underlying hematologic disease. Case Rep
Hematol. 2015;2015:180526.

8. Talano JA, Hillery CA, Gottschall JL, Baylerian DM, Scott JP. Delayed hemolytic transfusion reaction/hyperhemolysis syndrome in children with sickle cell disease. Pediatrics. 2003;111(6 Pt 1):e661-665.

9. Win N, New H, Lee E, de la Fuente J. Hyperhemolysis syndrome in sickle cell disease: case report (recurrent episode) and literature review. Transfusion. 2008;48(6):1231-1238.

10. Islam MS, Chia L. Hyperhemolysis syndrome in a patient with sickle cell disease with erythrophagocytosis in peripheral blood. Eur J Haematol. 2010;84(2):188.

11. Gladwin MT. Revisiting the hyperhemolysis paradigm. Blood. 2015;126(6):695-696.

12. Gouveia ME, Soares NB, Santoro MS, de Azevedo FC. Hyperhemolysis syndrome in a patient with sickle cell anemia: case report. Rev Bras Hematol Hemoter. 2015;37(4):266-268.

13. Vlachaki E, Gavriilaki E, Kafantari K, Adamidou D, Tsitsikas D, Chasapopoulou E, Anagnostopoulos A, et al. Successful outcome of hyperhemolysis in sickle cell disease following multiple lines of treatment: the role of complement inhibition. Hemoglobin. 2018;42(5-6):339341.

14. Rothman SM, Nelson JS. Spinal cord infarction in a patient with sickle cell anemia. Neurology. 1980;30(10):10721076.

15. Edwards A, Clay EL, Jewells V, Adams S, Crawford RD, Redding-Lallinger R. A 19-year-old man with sickle cell disease presenting with spinal infarction: a case report. J Med Case Rep. 2013;7:210. 